\title{
Tuning the hopping parameter in the Oktay-Kronfeld action for charm and bottom quarks on a MILC HISQ ensemble
}

\author{
Hwancheol Jeong, Weonjong Lee, Jaehoon Leem, Sungwoo Park* \\ Lattice Gauge Theory Research Center, CTP, and FPRD, \\ Department of Physics and Astronomy, Seoul National University, Seoul, 151-747, South Korea \\ E-mail: wlee@snu.ac.kr
}

Tanmoy Bhattacharya, Rajan Gupta, Yong-Chull Jang

T-2, Theoretical Division,

Los Alamos National Laboratory, Los Alamos, NM 87545, USA

E-mail: rgelanl.gov

\section{LANL/SWME Collaboration}

The first step in the calculation of semi-leptonic form factors in the decay of heavy mesons is the tuning of the hopping parameter $\kappa$ for the charm and bottom quark masses. Results for the Oktay-Kronfeld (OK) action are presented for one $N_{f}=2+1+1$ HISQ ensemble generated by the MILC collaboration at $a \approx 0.12 \mathrm{fm}$ and $M_{\pi} \approx 310 \mathrm{MeV}$. Estimates of hyperfine splitting of heavy-light and heavy-heavy mesons are presented and the inconsistency parameter is evaluated.

34th Annual International Symposium on Lattice Field Theory

24-30 July 2016

University of Southampton, UK

\footnotetext{
* Speaker.
} 


\section{Introduction}

There are two independent methods to extract $V_{c b}$ with $B$-meson decays. One is the heavy quark expansion method based on QCD sum rules with inclusive $B$-meson decays: $\bar{B} \rightarrow X_{c} \ell \bar{v}$, and the other is the lattice QCD method to calculate the semileptonic form factors in the analysis of the exclusive $B$-meson decays: $\bar{B} \rightarrow D^{(*)} \ell \bar{v}$. There exists about $3 \sigma$ tension in $\left|V_{c b}\right|$ between the inclusive and exclusive decay channels [1,2]. The future experiment at KEK (Belle 2) will increase statistics for $B$-meson decays dramatically (by a factor of 50). It is time to improve the lattice results of semileptonic form factors for the exclusive $B$-meson decays. Since the dominant error in lattice QCD results for $\left|V_{c b}\right|$ comes from the heavy quark discretization, we simulate the Oktay-Kronfeld (OK) action [3], a highly improved version of the Fermilab formulation.

If we use the OK action instead of the clover action (the original action of the Fermilab formulation [4]), then the power counting estimate suggests that the discretization error due to charm quarks can be reduced from $1.0 \%$ (clover) down to $0.2 \%$ (OK) for the semileptonic form factor for the $\bar{B} \rightarrow D^{*} \ell \bar{v}$ decay at zero recoil. The OK action is improved to $\mathscr{O}\left(\lambda^{3}\right)$ in HQET power counting, and $\mathscr{O}\left(v^{6}\right)$ in NRQCD power counting, while the clover action is improved to $\mathscr{O}\left(\lambda^{2}\right)$ in HQET and to $\mathscr{O}\left(v^{4}\right)$ in NRQCD. One drawback is that the OK action takes significantly more computing resources (by a factor of $\approx 50$ ) to calculate its propagator. We measured heavy-light (HL) and heavy-heavy $(\mathrm{HH})$ meson spectra to probe the improvement by the OK action, and the inconsistency parameter and hyperfine splitting showed clear improvement [5].

In this paper, we tune hopping parameters using the physical $B_{s}$ and $D_{s}$ meson spectrum on the coarse MILC HISQ ensemble at $a \approx 0.12 \mathrm{fm}$.

\section{Simulation Details}

We use the coarse ( $a \approx 0.12 \mathrm{fm}$ ) ensemble of the MILC HISQ lattices [6]. The lattice geometry is $24^{3} \times 64$. The tadpole improvement coefficient is $u_{0}=0.86372$ from the plaquette Wilson loop. The sea quark masses are $a m_{\ell}=0.0102$ for light quarks, $a m_{s}=0.0509$ for the strange quark, and $a m_{c}=0.635$ for the charm quark. For the HL mesons, $B_{s}^{(*)}$ and $D_{s}^{(*)}$, we use the HISQ action for the strange quark, and the OK action for charm and bottom quarks. Heavy quark propagators are generated using an optimized BiCGStab inverter [7]. In the OK action, the tadpole improved bare quark mass $m_{0}$ is related to the hopping parameter $\kappa$ as follows,

$$
a m_{0}=\frac{1}{2 u_{0} \kappa}-\left(1+3 \zeta r_{s}+18 c_{4}\right)
$$

where $c_{4}$ is the tree-level matching coefficient of a dimension-7 operator in the OK action [3]. Here, we set $\zeta=1$ for isotropic lattices and $r_{s}=1$ as is standard for the Wilson clover action. To tune the hopping parameter $\kappa$ to the physical values, we simulate four $\kappa$ values each for the bottom and the charm quarks as shown in Table 1. The parameters of covariant Gaussian smearing used at both the source and sink of heavy quark propagators to reduce the excited state contamination [8] are given in Table 1. For HISQ valence quarks, we use point source and sink.

We calculate $\mathrm{HL}$ and $\mathrm{HH}$ meson correlators on 500 configurations using 6 sources for bottom quarks and 3 sources for charm quarks. We use jackknife resampling to estimate the statistical 


\begin{tabular}{cc}
\hline \hline$a m_{s}^{v}$ & $\varepsilon$ \\
\hline 0.0509 & -0.0017468 \\
\hline \hline
\end{tabular}

(a) HISQ parameters

\begin{tabular}{cccc}
\hline \hline$\kappa$ (bottom) & $\kappa($ charm $)$ & $\sigma$ & $N_{G S}$ \\
\hline $0.042,0.041,0.040,0.039$ & $0.049,0.0487,0.0483,0.048$ & 5 & 60 \\
\hline \hline
\end{tabular}

(b) Tadpole improved OK parameters

Table 1: Parameters used for generating the valence quark propagators. (a) $m_{s}^{v}$ is set to the physical strange quark mass, and $\varepsilon$ is the coefficient of the Naik term in the HISQ action [6]. (b) $\kappa$ values for the bottom and charm quarks. The covariant Gaussian smearing parameters $\sigma$ and $N_{G S}$ are defined in Ref. [8].

error. We fix the time separation between sources to $\Delta t=6$. We choose the initial source time slice randomly for each configuration. We use 11 different momentum projections for the two-point meson correlation functions. To increase the statistics, we use the time reflection symmetry of the two-point correlation functions.

\section{Fits to the Meson Correlators and the Dispersion Relation}

The numerical data for the two-point meson correlators is fit using

$$
\begin{aligned}
& f^{\mathrm{HL}}(t ; \mathbf{p})=A e^{-E t}\left(1-(-1)^{t} r e^{-\Delta E t}\right)+A e^{-E(T-t)}\left(1-(-1)^{t} r e^{-\Delta E(T-t)}\right) \\
& f^{\mathrm{HH}}(t ; \mathbf{p})=A e^{-E t}+A e^{-E(T-t)} .
\end{aligned}
$$

The HL meson correlator, $f^{\mathrm{HL}}$, has 4 fit parameters: the ground state energy and amplitude $(E$, $A)$, an amplitude ratio $\left(r=A^{p} / A\right)$, and energy difference $\left(\Delta E=E^{p}-E\right)$, where the superscript $p$ stands for the opposite parity partner state that is present in staggered fermion correlation functions. $f^{\mathrm{HH}}$ is the function used to fit the HH mesons. The range $12 \leq t \leq 19$ is used to fit the HL mesons and $12 \leq t \leq 16$ for the HH mesons. The ground state energy $E(\mathbf{p})$ is then fit using the following dispersion relation:

$$
E(\mathbf{p})=M_{1}+\frac{\mathbf{p}^{2}}{2 M_{2}}-\frac{\left(\mathbf{p}^{2}\right)^{2}}{8 M_{4}^{3}}-\frac{a^{3} W_{4}}{6} \sum_{i=1}^{3} p_{i}^{4},
$$

to obtain $M_{1}$ the rest mass, $M_{2}$ the kinetic mass, $M_{4}$ the quartic mass, and $W_{4}$ the Lorentz symmetry breaking term. In both fits we use the full covariance matrix with trivial priors.

\section{Kappa Tuning}

We determine the hopping parameters $\kappa_{b}$ and $\kappa_{c}$ such that the kinetic masses are equal to the physical $B_{s}$ and $D_{s}$ masses, respectively. We tune the kinetic mass $M_{2}$ rather than the rest mass $M_{1}$. The form factors and decay constants which we are interested in are independent of the rest mass $M_{1}$ in the Fermilab interpretation of improved Wilson fermions.

We use the HQET inspired fitting function for kinetic HL meson masses,

$$
a M_{2}(\kappa)=d_{0}+a m_{2}(\kappa)+\frac{d_{1}}{a m_{2}(\kappa)}+\frac{d_{2}}{\left(a m_{2}(\kappa)\right)^{2}},
$$

where $M_{2}$ is the kinetic mass of the HL meson, and $m_{2}$ is the kinetic mass of the heavy quark. We determine $d_{0}, d_{1}$ and $d_{2}$ using the correlated least $\chi^{2}$ fitting. Here, $m_{2}(\kappa)$ is related to the bare mass 

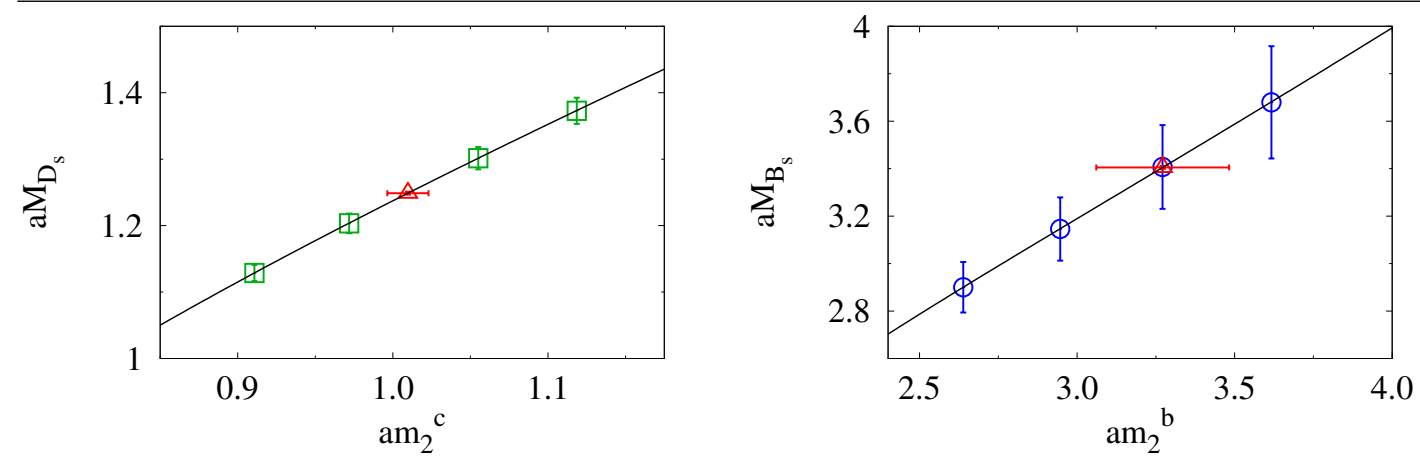

Figure 1: Plot of the pseudoscalar meson mass $D_{s}\left(B_{s}\right)$ versus the kinetic quark mass $m_{2}^{c}\left(m_{2}^{b}\right)$ defined in Eq. (4.2). The physical $\kappa_{c}$ and $\kappa_{b}$ (shown as red triangles and given in Table 2) are determined by tuning the $D_{S}$ and $B_{S}$ pseudoscalar masses to their experimental values.

$m_{0}(\kappa)$ at the tree level as follows,

$$
\frac{1}{a m_{2}}=\frac{2 \zeta^{2}}{a m_{0}\left(2+a m_{0}\right)}+\frac{r_{s} \zeta}{1+a m_{0}}
$$

Figure 1 shows the interpolation of $m_{2}$ to the physical values for the bottom and charm quarks. Results for $\kappa_{b}$ and $\kappa_{c}$ are summarized in Table 2. In Table 2, we present the $\kappa$-tuning results using physical values of the pseudoscalar meson mass $\left(M_{X}\right)$, vector meson mass $\left(M_{X^{*}}\right)$, and the spinaveraged mass $\left(M_{X}+3 M_{X^{*}}\right) / 4$ for $X=B_{s}$ or $D_{s}$. We find that all the results for $\kappa$ determined from different spin states are consistent within statistical uncertainty. We also perform another fit using a simpler fitting function: $a M_{2}=d_{0}+a m_{2}+d_{1} /\left(a m_{2}\right)$, and take the difference in $\kappa$ as the systematic error due to ambiguity in the fitting function.

\begin{tabular}{llll}
\hline \hline $\mathrm{X}$ & $M_{B_{s}}^{X}(\mathrm{GeV})$ & $a M_{B_{s}}^{X}$ & $\kappa_{b}$ \\
\hline pseudoscalar & $5.36682(22)$ & $3.4051(61)$ & $0.04000(63)(2)(2)$ \\
vector & $5.4154_{-0.0021}^{+0.0024}$ & $3.4360_{-0.0074}^{+0.0076}$ & $0.03932(90)(3)(3)$ \\
spin-average & $5.4033_{-0.0016}^{+0.0019}$ & $3.4283_{-0.0071}^{+0.0072}$ & $0.03950(80)(3)(4)$ \\
\hline \hline $\mathrm{X}$ & $M_{D_{s}}^{X}(\mathrm{GeV})$ & $a M_{D_{s}}^{X}$ & $\kappa_{c}$ \\
\hline pseudoscalar & $1.96827(10)$ & $1.2488(23)$ & $0.048517(63)(9)(1)$ \\
vector & $2.1121(4)$ & $1.3401(26)$ & $0.048281(163)(12)(2)$ \\
spin-average & $2.0761(3)$ & $1.3173(25)$ & $0.048346(126)(11)(3)$ \\
\hline \hline
\end{tabular}

Table 2: Results of tuning the $\kappa$ for the bottom $\left(\kappa_{b}\right)$ and charm $\left(\kappa_{c}\right)$ quarks. For converting the experimental $M$ to $a M$, we use $a=0.12520(22) \mathrm{fm}$ [9] . In $\kappa_{b, c}$, the first error is statistical, the second error is propagation of experimental error in $M^{X}$, and the third error is systematic to account for the uncertainty in the fit ansatz.

\section{Inconsistency Parameter}

The inconsistency parameter $I[10,11]$ is used to see $O\left(\mathbf{p}^{4}\right)$ improvement in the OK action. Let us use $Q$ for heavy quarks and $q$ for light quarks, and define $\delta M \equiv M_{2}-M_{1}$ as the difference 

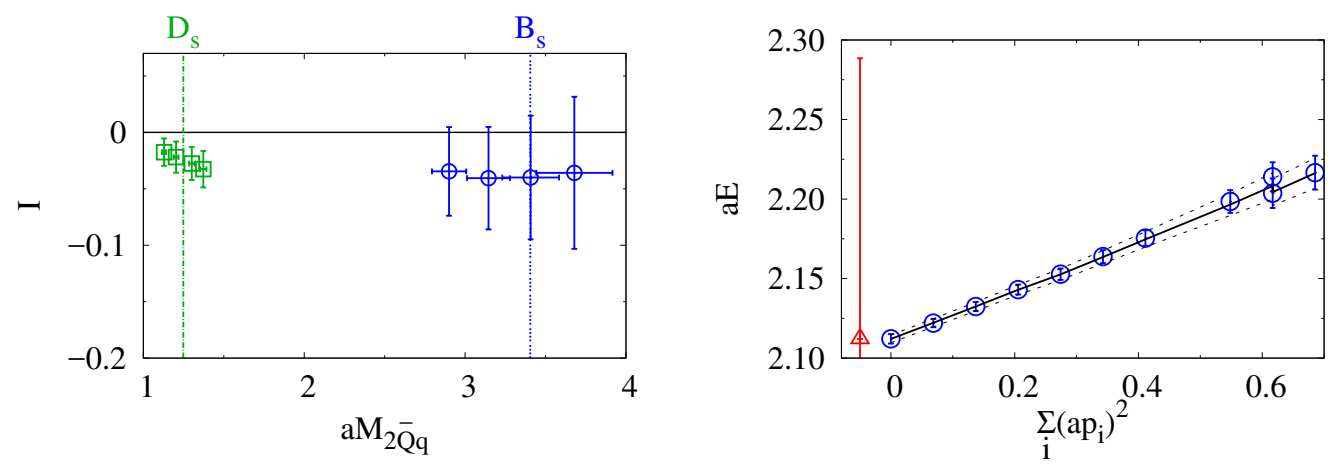

Figure 2: (Left) Inconsistency parameter $I$ versus the pseudoscalar mass $a M_{2 \bar{Q} q}$ for charm (green squares) and bottom (blue circles) quarks. (Right) Dispersion relation for the HL bottom meson with $\kappa=0.040$. The rest mass, $M_{1}=2.112(2)$, is given by the blue circle at $\boldsymbol{p}=0$. The kinetic mass, $M_{2}=3.408(176)$, is extracted from the fit and shown by the red triangle translated to $E=2.112$. Note that the determination of the error in $M_{2}$ is much larger than in $M_{1}$.

between the kinetic and rest masses. Then the inconsistency parameter $I$ is

$$
I \equiv \frac{2 \delta M_{\bar{Q} q}-\left(\delta M_{\bar{Q} Q}+\delta M_{\bar{q} q}\right)}{2 M_{2 \bar{Q} q}}=\frac{2 \delta B_{\bar{Q} q}-\left(\delta B_{\bar{Q} Q}+\delta B_{\bar{q} q}\right)}{2 M_{2 \bar{Q} q}}
$$

Here the binding energies $B_{1,2}$ are

$$
M_{1 \bar{Q} q}=m_{1 \bar{Q}}+m_{1 q}+B_{1 \bar{Q} q}, \quad M_{2 \bar{Q} q}=m_{2 \bar{Q}}+m_{2 q}+B_{2 \bar{Q} q}
$$

for HL mesons. Here the quark masses $m_{1,2}$ are defined by the quark dispersion relation, which is similar to Eq. (3.3). We neglect the light quarkonium contribution $\delta M_{\bar{q} q}$ (and $\delta B_{\bar{q} q}$ ). In Fig. 2 we present results for $I$ for pseudoscalar mesons. Near the $B_{s}$ region, $I$ is consistent with the continuum limit, $I=0$, within the error bars, which indicates a dramatic improvement from that of the Fermilab action: $I \approx-0.6[5]$.

\section{Hyperfine Splittings}

We define the hyperfine splittings of HL and HH pseudoscalar mesons, $\Delta_{1}$ and $\Delta_{2}$ as

$$
\Delta_{1}=M_{1}^{*}-M_{1}, \quad \Delta_{2}=M_{2}^{*}-M_{2},
$$

and plot $\Delta_{2}$ versus $\Delta_{1}$ in Fig. 3. As illustrated in Fig. 2, $M_{2}$ has much larger errors than $M_{1}$ since it is extracted from the slope versus momentum. Consequently, $\Delta_{2}$ has larger errors than $\Delta_{1}$.

The HQET expansion for $\Delta_{1}$ in the HL meson system is given in Ref. [12]:

$$
\Delta_{1}=M_{1}^{*}-M_{1}=\frac{4 \lambda_{2}}{2 m_{B}}-\frac{4 \rho_{2}}{4 m_{E}^{2}}+\frac{8 T_{2}}{2 m_{2} 2 m_{B}}+\frac{4\left(T_{4}-T_{2}\right)}{4 m_{B}^{2}}+\mathscr{O}\left(\frac{1}{m^{3}}\right),
$$

where $\lambda_{2}, \rho_{2}, T_{2}, T_{4}$ are HQET matrix elements defined in Ref. [12]. For the OK action, the matching conditions are $m_{2}=m_{B}=m_{E}$ [3]. Thus, $\Delta_{1}$ defined in Eq. (6.2) is in terms of the kinetic 


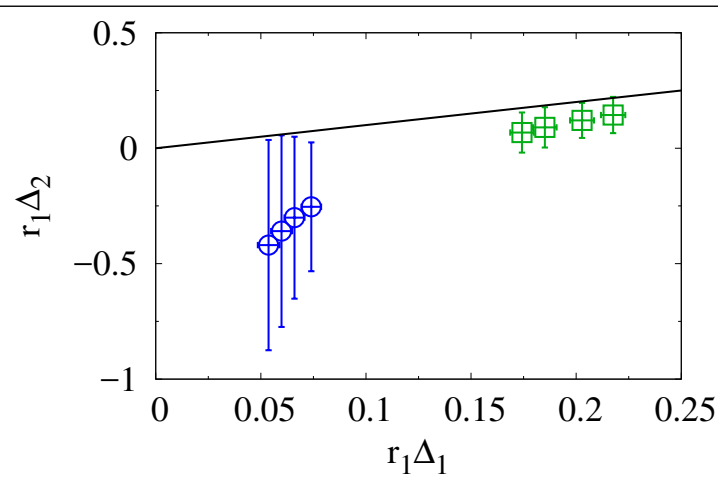

(a) Hyperfine splitting for HL mesons

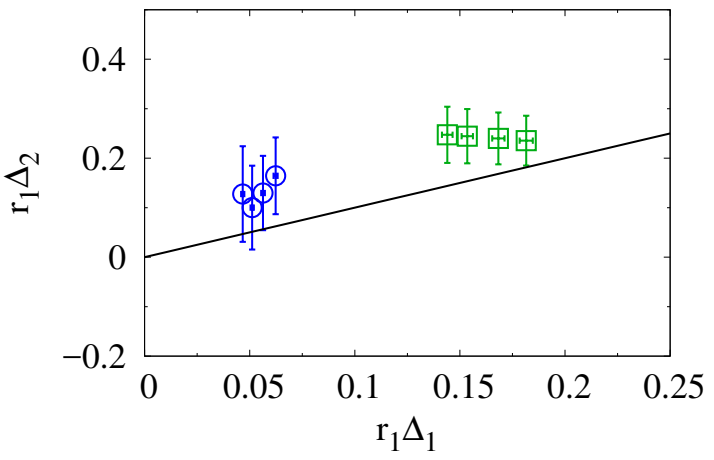

(b) Hyperfine splitting for $\mathrm{HH}$ mesons

Figure 3: Hyperfine splitting $\Delta_{2}$ versus $\Delta_{1}$ for the HL (Fig. 3(a)) and the HH (Fig. 3(b)) mesons in units of $r_{1}$ taken from Ref. [6]. The black line represents the continuum result, $\Delta_{1}=\Delta_{2}$.

quark mass, which was used to tune the $\kappa$ to the physical value. To analyze $\Delta_{1}$, we recast Eq. 6.2 as

$$
a \Delta_{1}=h_{0}+\frac{h_{1}}{a m_{2}}+\frac{h_{2}}{\left(a m_{2}\right)^{2}}+\frac{h_{3}}{\left(a m_{2}\right)^{3}},
$$

where $h_{1}=2 a^{2} \lambda_{2}$ and $h_{2}=a^{3}\left(-\rho_{2}+T_{2}+T_{4}\right)$. Because we have only 4 data points, we set $h_{3}=0$ in the fits. Correlated fits, shown in Fig. 4 , give $h_{0}=0$ within statistical uncertainty, consistent with the theoretical prediction. Our results, with $h_{0}$ set to zero in the fits are summarized in Table 3 . The corresponding $h_{i}$ from fits to $\Delta_{2}$ were very poorly determined.

We are performing simulations at other values of the lattice spacing and quark mass in order to perform the continuum-chiral extrapolation and compare with the experimental value.

\begin{tabular}{lllllll}
\hline \hline type & $h_{1}$ & $h_{2}$ & $\lambda_{2}\left(\mathrm{GeV}^{2}\right)$ & $A\left(\mathrm{GeV}^{3}\right)$ & $\Delta_{1}(\mathrm{MeV})$ & $\Delta_{\exp }(\mathrm{MeV})$ \\
\hline$B_{s}$ & $0.075(15)$ & $0.002(35)$ & $0.093(19)$ & $0.01(14)$ & $36.4(3.6)$ & $48.6_{-1.6}^{+1.8}$ \\
$D_{s}$ & $0.0697(45)$ & $0.0065(33)$ & $0.0866(59)$ & $0.025(13)$ & $118.9(3.4)$ & $143.8 \pm 0.4$ \\
\hline \hline
\end{tabular}

Table 3: Hyperfine splittings, $\lambda_{2}$ and $A \equiv-\rho_{2}+T_{2}+T_{4}$ for the $D_{s}$ and $B_{s}$ mesons at the physical values of $\kappa_{c}$ and $\kappa_{b} . \Delta_{\text {exp }}$ is the experimental value [13].

\section{Summary and Plan}

We tuned the bottom and charm quark masses using physical values for $B_{s}^{(*)}$ and $D_{s}^{(*)}$ mesons. Estimates from fits to the pseudoscalar, vector, and spin-averaged mesons masses are consistent within their statistical uncertainty (see Table 2). We used estimates from the pseudoscalar mesons for the analysis of the hyperfine splittings. These values of $\kappa_{b}$ and $\kappa_{c}$ are now being used to measure the semileptonic form factors for the exclusive decays $\bar{B} \rightarrow D^{(*)} \ell \bar{v}$.

\section{Acknowledgments}

We thank Jon A. Bailey for helpful comments and suggestions. The research of W. Lee is supported by the Creative Research Initiatives Program (No. 20160004939) of the NRF grant funded 

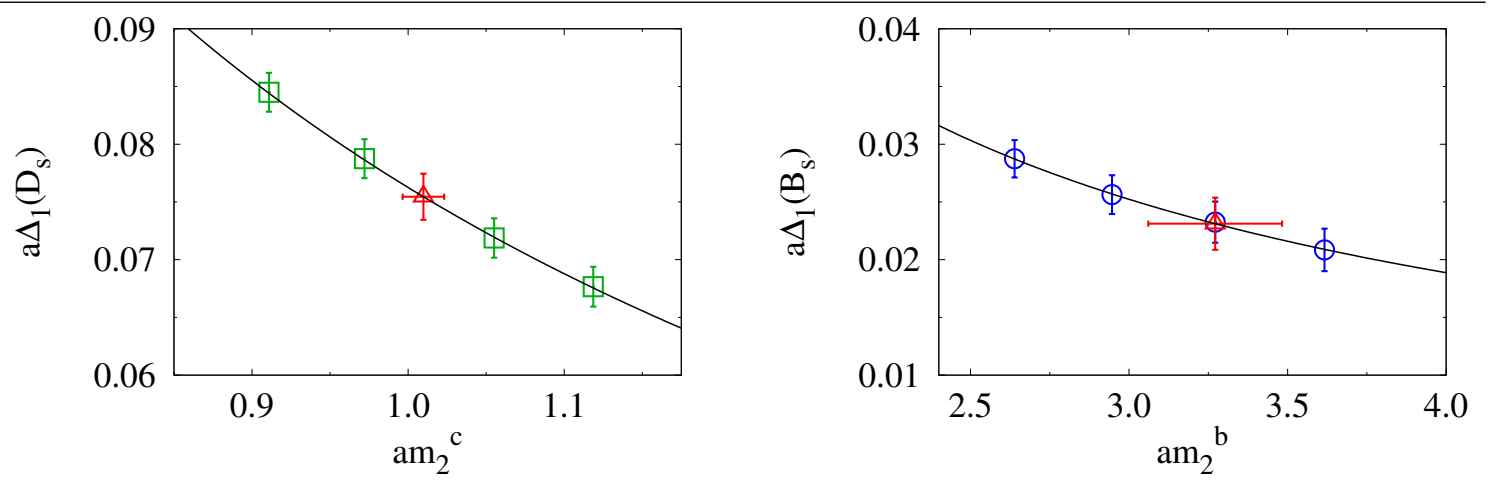

Figure 4: Plots of $\Delta_{1}\left(D_{s}\right)$ (left panel) and $\Delta_{1}\left(B_{s}\right)$ (right panel) versus the kinetic masses, $m_{2}^{c}$ and $m_{2}^{b}$, of the quarks. Results at the physical quark masses, tuned using the pseudoscalar meson masses, are shown by the red triangles and given in Table 3.

by the Korean government (MEST). W. Lee would like to acknowledge the support from the KISTI supercomputing center through the strategic support program for the supercomputing application research (No. KSC-2014-G2-002). Computations were carried out in part on the DAVID GPU clusters at Seoul National University. The research of T. Bhattacharya, R. Gupta and Y-C. Jang is supported by the U.S. Department of Energy, Office of Science of High Energy Physics under contract number DE-KA-1401020, the LANL LDRD program and Institutional Computing.

\section{References}

[1] SWME Collaboration, J. A. Bailey, Y.-C. Jang, W. Lee, and S. Park Phys. Rev. D92 (2015) 034510, [1503.05388].

[2] J. A. Bailey, W. Lee, J. Leem, S. Park, and Y.-C. Jang PoS LATTICE2016 (2016) 383, [1611.00503].

[3] M. B. Oktay and A. S. Kronfeld Phys. Rev. D78 (2008) 014504, [0 803 . 0 523].

[4] A. X. El-Khadra, A. S. Kronfeld, and P. B. Mackenzie Phys. Rev. D55 (1997) 3933-3957, [hep-lat/9604004].

[5] Fermilab Lattice, SWME, MILC Collaboration, J. A. Bailey, Y.-C. Jang, W. Lee, C. DeTar, A. S. Kronfeld, and M. B. Oktay PoS LATTICE2015 (2016) 099, [1 601 . 0475 9].

[6] MILC Collaboration, A. Bazavov et al. Phys. Rev. D87 (2013), no. 5 054505, [1212 . 4768 ].

[7] Fermilab Lattice, SWME, MILC Collaboration, Y.-C. Jang, J. A. Bailey, W. Lee, C. DeTar, M. B. Oktay, and A. S. Kronfeld PoS LATTICE2013 (2014) 030, [1311. 5029].

[8] B. Yoon et al. Phys. Rev. D93 (2016), no. 11 114506, [1602 . 07737].

[9] Fermilab Lattice, MILC Collaboration, A. Bazavov et al. Phys. Rev. D90 (2014), no. 7 074509, [1407.3772].

[10] S. Collins, R. G. Edwards, U. M. Heller, and J. H. Sloan Nucl. Phys. Proc. Suppl. 47 (1996) 455-458, [hep-lat/9512026].

[11] A. S. Kronfeld Nucl. Phys. Proc. Suppl. 53 (1997) 401-404, [hep-lat/9608139].

[12] A. S. Kronfeld Phys. Rev. D62 (2000) 014505, [hep-lat / 0002008 ].

[13] K. A. Olive Chin. Phys. C40 (2016), no. 10100001. 